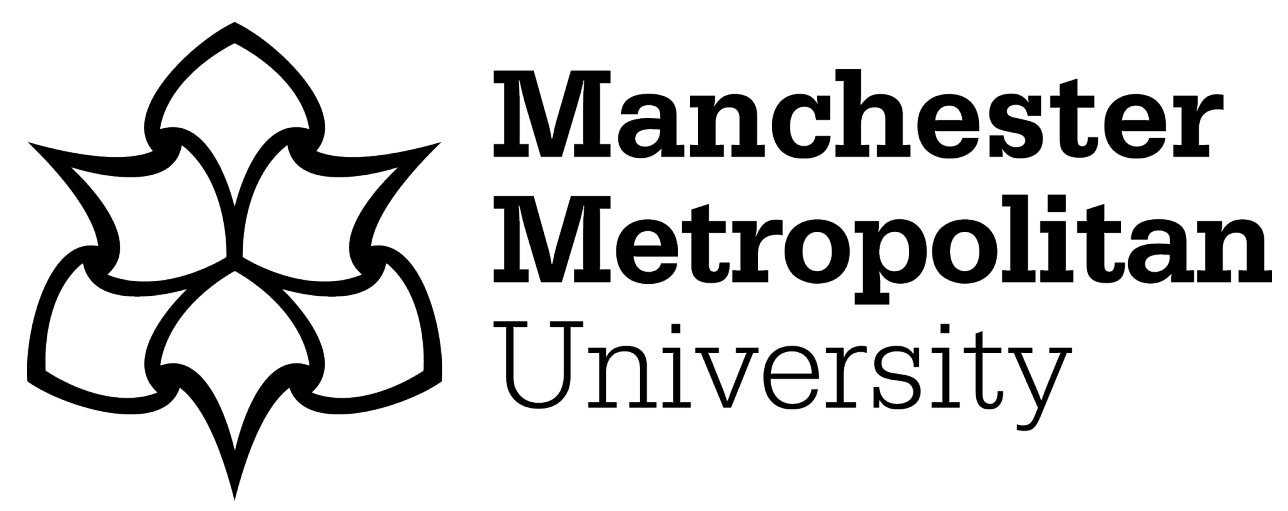

Salmon, NA ORCID logoORCID: https://orcid.org/0000-0003-4786-7130 (2016) Experimental results and simulations from aperture synthesis threedimensional radiometric imaging. In: SPIE Security and Defence, 26 September 2016 - 29 September 2019, Edinburgh, Scotland.

Downloaded from: https://e-space.mmu.ac.uk/623610/

Version: Published Version

Publisher: SPIE

DOI: https://doi.org/10.1117/12.2231696

Please cite the published version 


\title{
Experimental results and simulations from aperture synthesis three- dimensional radiometric imaging
}

\author{
Neil A. Salmon \\ Manchester Metropolitan University, Manchester, M15 6BH, UK
}

\begin{abstract}
This paper presents the theory and algorithm of how a three-dimensional (3D) image can be generated using crosscorrelations of radiometric emission from a source measured using antennas in the near field. An example of how the algorithm is used to create 3D images of emission measured from a noise source is presented, indicating the presence of Fresnel noise and aliasing in the experimental data when the source is moved away from the phase centre. Simulations are presented which reproduce the Fresnel noise as generated by a $3 \times 3 \times 3$ array of point sources located at the centre of a 2 metre diameter array of antennas representing a security screening portal. Two methods of reducing the Fresnel noise are presented: 1) a software method which makes successive more accurate estimates of the locations and intensities of sources; 2) a hardware method which reduces the coherence length of the radiation by increasing the radiation bandwidth.
\end{abstract}

Keywords: Aperture synthesis, imaging, tomography, three-dimensional, millimetre wave, security, near-field, Fresnel scale

\section{INTRODUCTION}

The van Cittert Zernike theorem ${ }^{[3]}$ underpins radio astronomy aperture synthesis, a technique which has been used for decades to create two-dimensional (2-D) images of astrophysical subjects ${ }^{[4]}$. It has been demonstrated that this offers a route to $2 \mathrm{D}$ imaging in the near-field for applications of passive millimetre wave imaging for security screening and allweather capabilities ${ }^{[2]}$. Recently an algorithm based on an extension of the aperture synthesis technique to threedimensions was shown by simulation to be capable of generating three-dimensional (3-D) images ${ }^{[1]}$. These simulations of point sources indicated that the spatial resolution possible in three-dimensions was half the wavelength of the radiation that was measured. This indicates that high resolution images can be generated in potential security screening portals by operating at relatively low frequencies in the millimetre wave band, thus minimising system costs. Subsequent work indicates when sources of moved approximately a Fresnel scale away from the phase centre of the 3D image Fresnel 'noise' is generated in the image ${ }^{[5]}$. This is not true noise but an artefact of the processing associated with a break down in the Fourier transform relationship between the visibility function (formed from the cross-correlations) and the 3D image. This work demonstrates the use of the basic algorithm on experimental data and simulations of a technique to reduce the effects of Fresnel noise.

\section{THREE-DIMENSIONAL IMAGE GENERATION THEORY}

The generalised van Cittert Zernike theorem is a Fourier transform relationship between the three-dimensional distribution of radiometric sources $I(\mathbf{l})$, where $\mathbf{l}$ is the position vector of the $3 \mathrm{D}$ image voxel from a phase centre, and a three-dimensional visibility function $V(\mathbf{u})$, where $\mathbf{u}$ is the spatial frequency position vector, as given in Eq. 1. The antenna response is given by $\mathrm{A}(\mathbf{l})$.

$$
I(\mathbf{l})=[A(\mathbf{l})]^{-1} \int_{\mathbf{u}_{M I N}}^{\mathbf{u}_{M A X}} V(\mathbf{u}) \exp (i \mathbf{u} . \mathbf{l}) d^{3} u
$$

* Correspondence: Email: n.salmon at mmu.ac.uk; Telephone: +447921172892 
The three-dimensional visibility function is created from the cross-correlations $V_{J, K}^{\prime}$ of electric fields $\left(v_{\mathrm{J}}\right.$ and $\left.v_{\mathrm{K}}\right)$, measured at pairs $(J, K)$ of antennas, as indicated in Eq. 2. The cross-correlation $V_{J, K}^{\prime}$ is a complex quantity containing phase and amplitude information. There are $n(n-1) / 2$ cross-correlations for a collection of $n$ antennas and these contain all of the $3 \mathrm{D}$ information about the subject.

$$
V_{J, K}^{\prime}=\left\langle v_{J}(t) v_{K}^{*}(t)\right\rangle
$$

The correlations need to be phase calibrated to define the centre of the 3D image. This can be performed experimentally by measuring the cross-correlations from a point noise source, extracting the phases from this, $\phi_{\mathrm{J}, \mathrm{K}, \mathrm{CAL}}$, then phase shifting the signal cross-correlations by that amount, as indicated in Eq. 3. This puts the centre of the 3D image at the phase centre as defined by the position of the noise source. The phase calibration may also be performed by measuring the cross-correlations arising from radiometric emission exiting the antennas of the individual receivers in the array, a practical point source of radiation. From this information single set of correlations from the portal may be phase calibrated for multiple adjacent volumes in the portal, enable the complete volume in the portal to be imaged with a half wavelength resolution in 3D space.

$$
V_{J, K}\left(\mathbf{u}_{J, K}\right)=V_{J, K}^{\prime} \exp \left(-i \phi_{J, K, C A L}\right)
$$

The spatial frequency position vector $\mathbf{u}_{J, K}$ is the change of phase of the cross-correlations divided by the change in position of a radiometric source at the phase centre, as given by Eq. 4, ie a vector field derived ${ }^{[6]}$ from a scaler phase field $\phi_{J, K}$ by using the gradient operator, $\nabla$.

$$
\mathbf{u}_{J, K}=\nabla \phi_{J, K}
$$

From geometrical considerations of the antenna phase centre locations $\mathbf{R}_{\mathrm{J}}$ and $\mathbf{R}_{\mathrm{K}}$, and the image phase centre location $\mathbf{R}_{\mathrm{PC}}$, the spatial frequency vector is given by Eq. 5 .

$$
\mathbf{u}_{J, K}=\frac{2 \pi}{\lambda}\left(\frac{\mathbf{R}_{J}-\mathbf{R}_{P C}}{\left|\mathbf{R}_{J}-\mathbf{R}_{P C}\right|}-\frac{\mathbf{R}_{K}-\mathbf{R}_{P C}}{\left|\mathbf{R}_{K}-\mathbf{R}_{P C}\right|}\right)
$$

The 3D visibility function of Eq. 1 can then be formed by placing the phase calibrated cross-correlations $V_{J, K}$ at the spatial frequencies defined by the spatial frequency vector $\mathbf{u}_{J, K}$, thus forming a 3D function. Since the image is a real function by definition, the conjugate points of the cross-correlations can also be added to the visibility function, doubling the number of points and guaranteeing a real functioned 3D image when the Fourier transform in Eq. 1 is taken.

\section{EXPERIMENTAL RESULTS}

The effects of using the above algorithm on data taken with a $22 \mathrm{GHz} 32$-channel receiver array can be seen in Figure1. Here a noise source is moved across the field of view at a distance of $15 \mathrm{~cm}$ from the array. The 3-D imaging algorithm forms a 3D image in the volume of space in front of the array. A horizontal versus vertical slice of this 3D image is taken at a distance of $15 \mathrm{~cm}$ from the array and shown in the upper part of this figure. The lower part of this figure illustrates the horizontal versus perpendicular distance from the array. The data is phase calibrated by placing a noise source in the centre of the field of view. The right most images show the image of the noise source in that position. The image of the noise source is elongated in the direction away from the array. This is because the resolution in this direction is less than it is in the horizontal direction. As the source moves to the left, the reproduction of the image of the noise source becomes less clear, the centre image starts to show the appearance of Fresnel noise in both the horizontal-vertical image and the horizontal-range image. The image to the left illustrates the effect of aliasing and Fresnel noise to a greater extent.

Schemes to minimise the Fresnel noise are presented in the next section, whilst the effects of aliasing can be minimised by change the antenna layout to make it one of a random spatial orientation of antennas, as shown in earlier publications $^{[7]}$. 


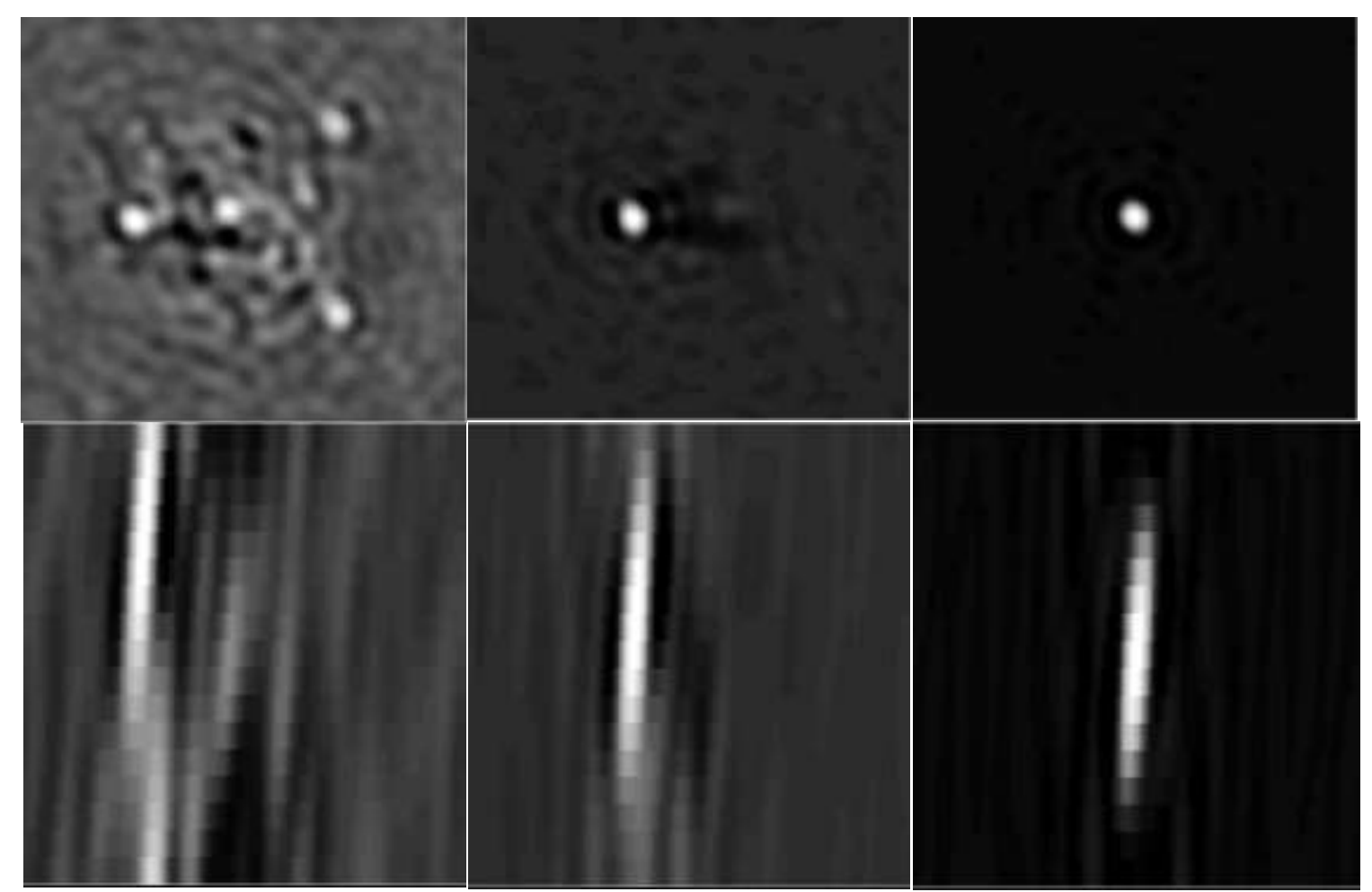

Figure 1: Experimental results from a noise source as it is moved from right to left, the upper images showing the central horizontal and vertical sections and the lower the horizontal and perpendicular distance sections from the 3-D image. The right most images corresponds to the source close to the phase centre, and the left most image at the extreme edge of the field of view, showing the effects of aliasing.
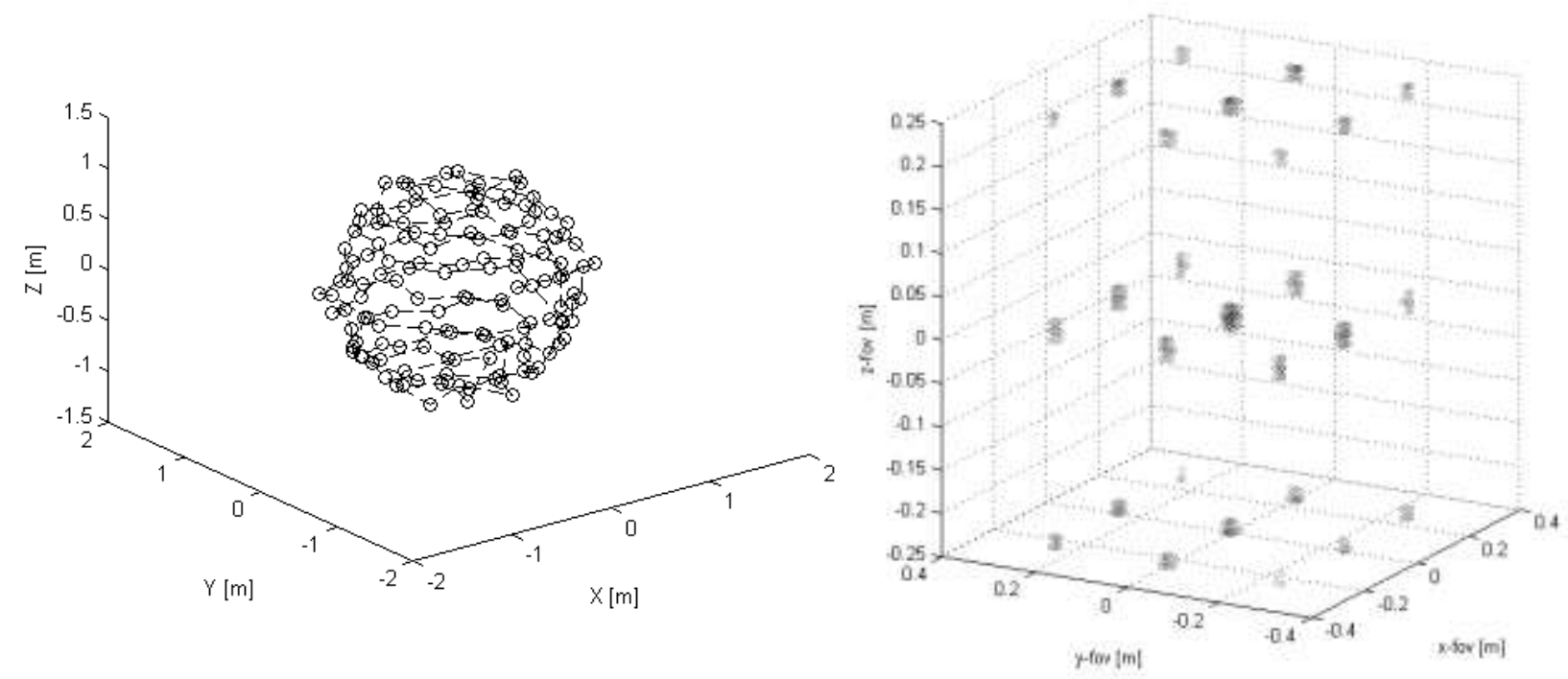

Figure 2. (a) The $2 \mathrm{~m}$ diameter spherical shell of antennas looking inward toward the sphere centre. (b) The $3 \times 3 \times 3$ array of point sources identified in the first iteration of Fresnel scale noise reduction algorithm. 


\section{SIMULATIONS OF ALGORITHMS TO REDUCED FRESNEL NOISE}

A 3-D array of point sources serves as a convenient subject to test the imaging algorithms through simulations. A $3 \times 3 \times$ 3 set of delta functions is a useful source for this purpose. The antenna array used in the simulation has a collection of

a)

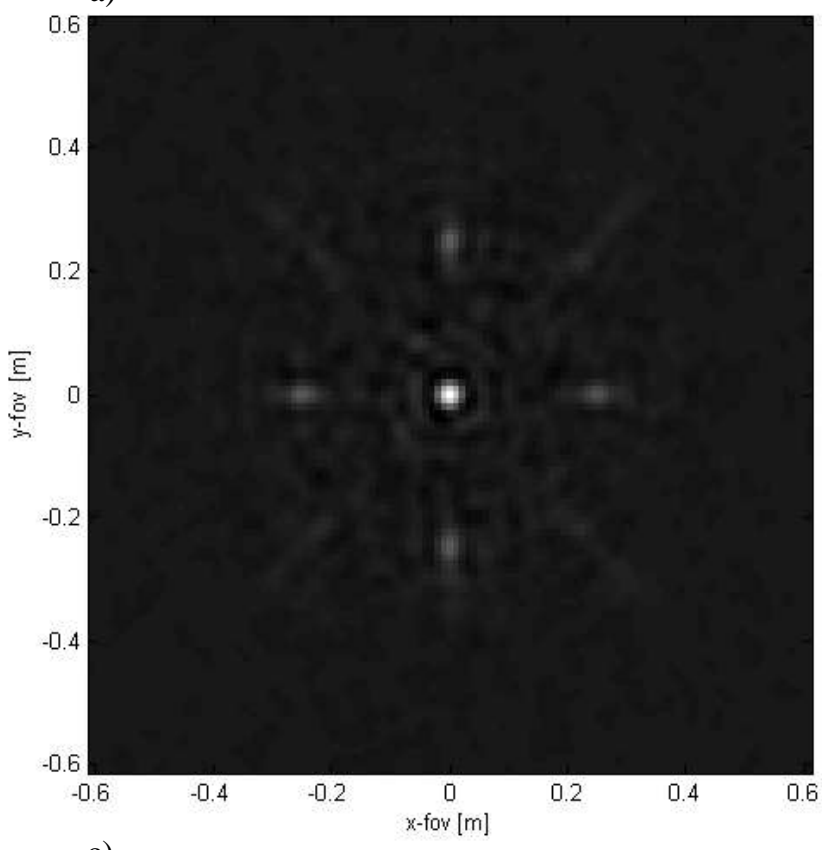

c)

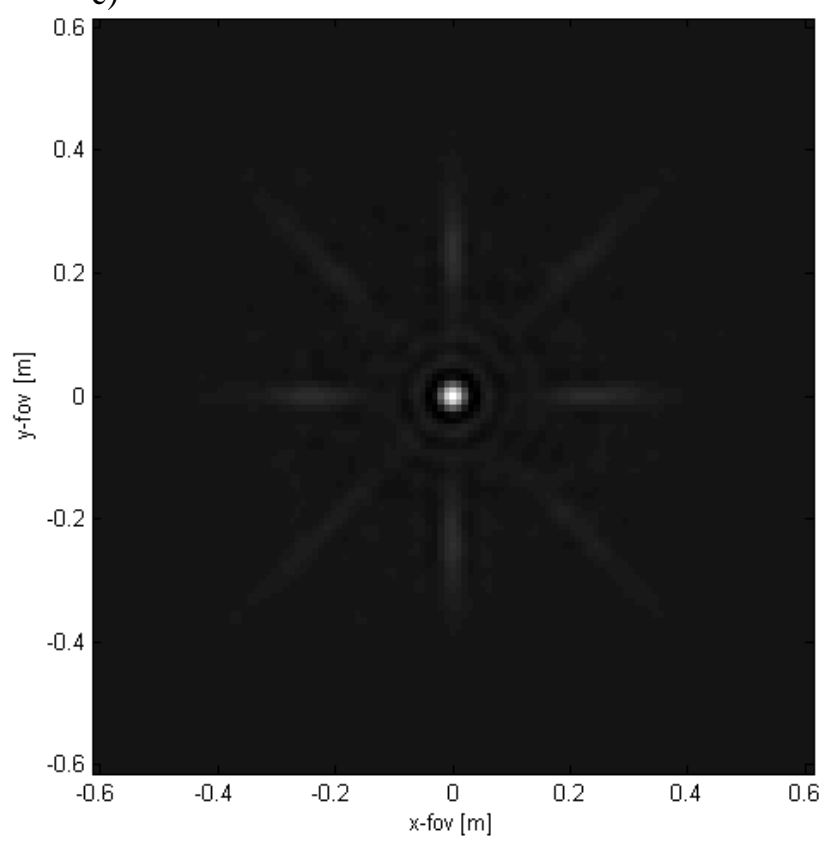

b)

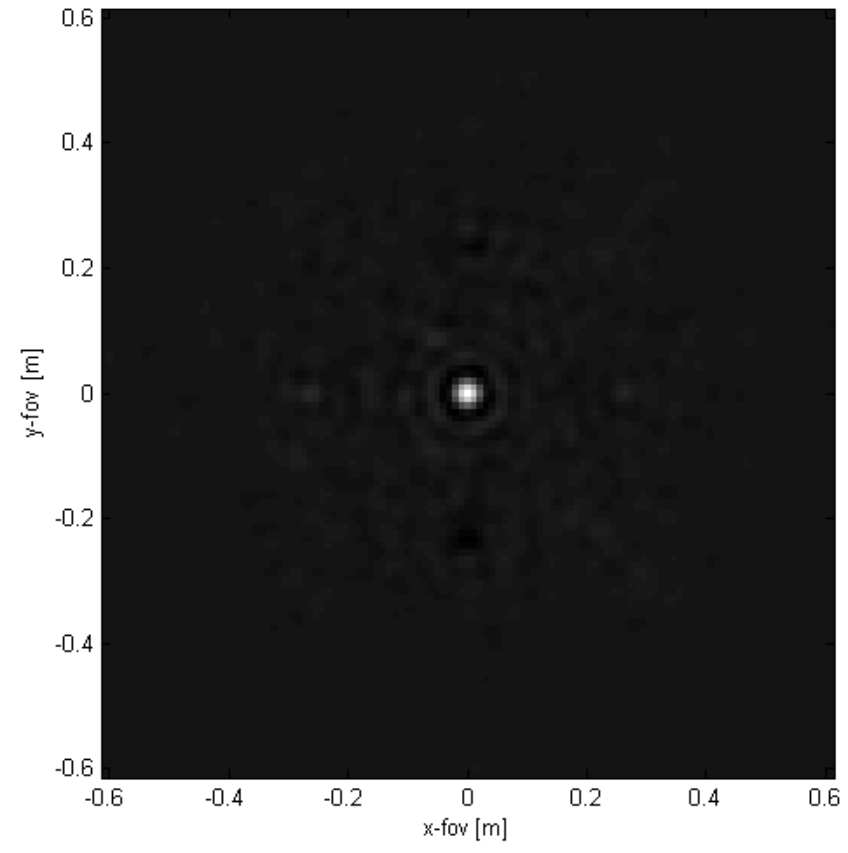

Figure 3. 2D image slices through the centre of the $3 \mathrm{D}$ image of the $3 \times 3 \times$ array of point sources. (a) Image formed by direct Fourier transform of the raw unprocessed visibility function. (b) Image formed by subtracting from the initial cross-correlations those cross-correlations generated by sources identified as being outside the Fresnel scale volume. (c) Image formed by increasing the radiation bandwidth from 300 $\mathrm{MHz}$ to $2 \mathrm{GHz}$, reducing coherence length to 15 $\mathrm{cm}$. Results in b) and c) indicate Fresnel noise can be minimised within a Fresnel scale around the phase centre.

147 low gain antennas on the surface of a sphere pointing towards the centre, sensitive at a frequency of $3.71 \mathrm{GHz}$ with a radiation bandwidth of $300 \mathrm{MHz}$, an architecture that might, funding permitting, be developed as a 3-D imaging demonstrator. The algorithm generates multiple 3-D images inside a two metre diameter sphere and the array of point sources is located at the centre, forming a cube having a side length of $50 \mathrm{~cm}$. The central slice of the 3D image of the array of point sources is shown in the top left of Figure 3. The central point source in the array is observable in the image 
centre, but the other sources are not, as at a distance from the centre of $25 \mathrm{~cm}$ they are close to the Fresnel scale, $\sqrt{ }(\lambda \mathrm{R}) \sim$ $28 \mathrm{~cm}$, where $\mathrm{R}$ is the distance to the phase centre, $\sim 1 \mathrm{~m}$. These sources also corrupt to image around the central region and this corruption is referred to at the Fresnel noise.

A software method of removing the Fresnel noise corruption is to create an approximate 3-D image of all the sources in the $2 \mathrm{~m}$ sphere by creating images of Fresnel scale volumes throughout the complete imaging volume. Such an image is displayed in $3 \mathrm{D}$ plot of the $3 \times 3 \times 3$ sources in Figure $2 \mathrm{~b}$ and demonstrates that simple processing of data using the algorithm of Sec. 2 is effective at identifying the locations of the brightest sources in a 3D scene.

This first 3D image can then be used to identify sources outside a particular Fresnel scale volume. This is used then to calculate a set of cross-correlations for these identified sources outside the Fresnel scale volume. These are then subtracted from the original set of cross-correlations, thus producing a set of cross-correlations from sources only within the Fresnel scale volume. These are then used to build the visibility function, which is Fourier transformed to generate an improved image of the Fresnel scale volume.

A $2 \mathrm{D}$ slice through the centre of the image for the volume centred on the central source of the $3 \times 3 \times 3$ array is shown in Figure $3 \mathrm{~b}$. This can be repeated for all Fresnel scale volume within the $2 \mathrm{~m}$ diameter sphere, thus generating an improved image of the full 3D volume. This can then be used to make a second iteration of a more accurate image. More work is needed to optimise the algorithm, such as finding the level of cross-correlations to remove from the original set of crosscorrelations and a more accurate determination of the locations of sources which corrupt the visibility function.

A hardware method of avoiding altogether the Fresnel noise is to increase the radiation bandwidth, $\mathrm{B}_{\mathrm{RF}}$. Increasing the bandwidth shortens the coherence length $l_{C} \sim c / B_{R F}$. This means if the path length difference from pairs of receivers differs by more than the coherence length there will be no correlation and so there will be no corruption of the visibility function from sources outside the Fresnel scale. Ideally the coherence length should be made to be of the order of the Fresnel scale. Increasing the radiation bandwidth to $2 \mathrm{GHz}$ shortens the coherence length to $15 \mathrm{~cm}$ resulting in the lower image of Figure 3. The Fresnel noise is reduced around the image of the central point source. However, if sources in other regions within the $2 \mathrm{~m}$ diameter sphere need to be imaged (digital) time delays would need to be introduced prior to cross-correlation and difference sets of cross-correlations would need to be collected for different Fresnel scale volumes within the $2 \mathrm{~m}$ diameter sphere, so images of the complete volume can be created.

\section{CONCLUSIONS AND FUTURE WORK}

The theory enabling 3D imaging of sources is presented. This is used on experimental data to demonstrate the technique can be used to image a noise source in 3-D. In this imagery the presents of Fresnel noise and aliasing can be seen. An algorithm to reduce the level of Fresnel noise is presented and works to first order. Optimisation of the algorithm is needed. Increasing the radiation bandwidth is also shown to be effective in reducing the level of Fresnel noise. Using this technique to image a full volume would require the introduction of time delays.

\section{REFERENCES}

[1] Salmon, N. A.,"3-D Radiometric Aperture Synthesis Imaging”, IEEE TMTT, Vol. 63, Issue 11, (2015).

[2] Salmon, N. A. Wilkinson, P.N. and Taylor, C., "Interferometric aperture synthesis for next generation passive millimetre wave imagers", SPIE Europe Security+Defence Europe, 'Millimetre Wave and Terahertz Sensors and Technology V', Edinburgh, September, (2012).

[3] Born, M, and Wolf, E., "Principles of optics", Cambridge University Press, 7th Edition, (2003).

[4] Thomson, A., Moran, M., Swenson, G, "Interferometry and Synthesis in Radio Astronomy", Wiley, (2004)

[5] Salmon, N. A. "Simulations of three-dimensional radiometric imaging of extended sources in a security screening portal", SPIE Europe Security+Defence Europe, "Millimetre Wave and Terahertz Sensors and Technology VIII, Toulouse, September 2015.

[6] Goodman, "Introduction to Fourier Optics", $3{ }^{\text {rd }}$ Ed., Roberts \& Company, (2005).

[7] Salmon, N. A., Wilkinson, P. and Taylor, C. 'Interferometric aperture synthesis for next generation passive millimetre wave images', SPIE Europe Security+Defence, Millimetre Wave Sensors and Technology, Edinburgh, September 2012. 